\title{
Response to letter to the editor: "comment on unplanned out-of-hospital birth and risk factors of adverse perinatal outcome: findings from a prospective cohort"
}

\author{
François Javaudin ${ }^{1,2}$, Arnaud Legrand ${ }^{3}$, Philippe Pes ${ }^{1}$, Emmanuel Montassier $^{1,2^{*}}$ (D) and Christelle Volteau ${ }^{3}$
}

\begin{abstract}
The aim of this Letter to the Editor was to respond to a comment highlighting potential statistical biases in an analysis of our recently published article. We therefore specified the method for selecting the model variables in order to limit overfitting, then we used the Firth method to control the sparse data bias, and finally for checking internal validity we used bootstrapping methods. In total, the conclusions of our model were not changed by these new analyses.
\end{abstract}

Keywords: Methodology, Multiple logistic regression, Firth method, Bootstrapping, Predictive performance

\section{To the Editor:}

We thank Dr. Yarandi and Tehrani [1] for their interest in our study [2] and to challenge us on specific statistical aspects. To avoid misinterpretations, we will try to clarify methodological trade-off we consent, and reveal complementary details and information of our dataset deep analysis.

Firstly, to limit overfitting, even if it was not explicitly described in the statistical part of the original paper, we indeed select predictor variables to input our multiple logistic regression model, on the basis of a stringent $p$-value $<0.2$. Looking at Table 2 of our original research, we can see that the selected variables defending a p-value between 0.03 and $<0.0001$.

Secondarily, we admit that we didn't process any penalization methods to control sparse data biases [3] on multiple pregnancy. But when we apply Firth method [4], model is strictly conserved and extremely wide interval remains tangible: Odds Ratio $=47.9$; Confidence Interval 95\%: 3.5-663.6. Moreover, even if multiple pregnancy, hypothermia, and/or materno-foetal infection can lead to circularity over-risk due to the imbrication of these clinical contexts with prematurity status, we rigorously explored intrinsic contribution and correlation and covariance

\footnotetext{
* Correspondence: emmanuelmontassier@hotmail.com

1 Department of Emergency Medicine, CHU Nantes, Nantes, France

${ }^{2}$ MiHAR lab, Université de Nantes, 44000 Nantes, France

Full list of author information is available at the end of the article
}

matrices. For example, Pearson chi2 test between "Prematurity" and "Hypothermia" highlights their independent predictive factor status ( $p$-value $=0.20$ ).

Finally, the predictive performance of model was internally validated through bootstrapping [5]. The multivariate model was refitted in 200 bootstraps samples (samples of the same size as the original population but with patients drawn randomly with replacement from the full study population) and then tested on the original sample. The measure of predictive discrimination used to characterize model performance, in both the original sample and the validation samples, was the area under the receiver operating characteristic curve (AUC). Average optimism, as the difference between bootstrap performance and test performance, was calculated. Optimism-corrected performance was then estimate by subtraction of the average optimism from the apparent performance (initial multivariate model). SAS software version 9.4 was used for validation. Initial multivariate model had an AUC of 0.78. In the 200 bootstrap samples, the mean AUC was 0.80 . When the models from each bootstrap sample were tested in the original sample, the AUC decreased to 0.75 . The optimism was then equal to 0.05 and the optimism-corrected AUC equal to 0.73 .

In total, despite the potential biases you mentioned, these results confirm our multivariate model as a first step exploratory analysis on morbi-mortality determinants in unplanned out-of-hospital birth. 


\section{Abbreviation}

AUC: Area under the receiver operating characteristic curve

\section{Acknowledgements}

Not applicable.

\section{Funding}

No funding.

\section{Availability of data and materials}

Please contact author for data requests.

\section{Authors' contributions}

This letter was written by FJ, AL, PP, EM and CV. AL and CV reperformed statistics. All authors read and approved the final manuscript.

\section{Ethics approval and consent to participate}

Not applicable.

\section{Consent for publication}

Not applicable.

\section{Competing interests}

The authors declare that they have no competing interests.

\section{Publisher's Note}

Springer Nature remains neutral with regard to jurisdictional claims in published maps and institutional affiliations.

\section{Author details}

${ }^{1}$ Department of Emergency Medicine, CHU Nantes, Nantes, France. ${ }^{2}$ MiHAR lab, Université de Nantes, 44000 Nantes, France. ${ }^{3}$ DRCI, CHU Nantes, Nantes, France.

Received: 30 April 2019 Accepted: 17 May 2019

Published online: 28 May 2019

\section{References}

1. Bidhendi Yarandi R, Panahi MH. Comment on unplanned out-of-hospital birth and risk factors of adverse perinatal outcome: findings from a prospective cohort. Scand J Trauma Resusc Emerg Med. 2019;27(1):37.

2. Javaudin F, Hamel V, Legrand A, Goddet S, Templier F, Potiron C, et al. Unplanned out-of-hospital birth and risk factors of adverse perinatal outcome: findings from a prospective cohort. Scand J Trauma Resusc Emerg Med. 2019;27(1):26.

3. Greenland S, Mansournia MA, Altman DG. Sparse data bias: a problem hiding in plain sight. BMJ. 2016;352:11981.

4. Firth D. Recent developments in quasi-likelihood methods. Bull Int Stat Inst. 1993:55:341-58.

5. Steyerberg EW. Clinical prediction models: a practical approach to development, validation, and updating: Springer Science \& Business Media; 2008. 